ARTICLE

https://doi.org/10.1038/s41467-019-09000-8

\title{
Reversible defect engineering in graphene grain boundaries
}

Krishna Balasubramanian ${ }^{1,6}$, Tathagatha Biswas ${ }^{2}$, Priyadarshini Ghosh 1,3,7, Swathi Suran ${ }^{1}$, Abhishek Mishra ${ }^{1}$ Rohan Mishra ${ }^{4}$, Ritesh Sachan (1) ${ }^{5,8}$, Manish Jain ${ }^{2}$, Manoj Varma', Rudra Pratap ${ }^{1} \&$ Srinivasan Raghavan ${ }^{1}$

Research efforts in large area graphene synthesis have been focused on increasing grain size. Here, it is shown that, beyond $1 \mu \mathrm{m}$ grain size, grain boundary engineering determines the electronic properties of the monolayer. It is established by chemical vapor deposition experiments and first-principle calculations that there is a thermodynamic correlation between the vapor phase chemistry and carbon potential at grain boundaries and triple junctions. As a result, boundary formation can be controlled, and well-formed boundaries can be intentionally made defective, reversibly. In $100 \mu \mathrm{m}$ long channels this aspect is demonstrated by reversibly changing room temperature electronic mobilities from 1000 to $20,000 \mathrm{~cm}^{2} \mathrm{~V}^{-1} \mathrm{~s}^{-1}$. Water permeation experiments show that changes are localized to grain boundaries. Electron microscopy is further used to correlate the global vapor phase conditions and the boundary defect types. Such thermodynamic control is essential to enable consistent growth and control of two-dimensional layer properties over large areas.

\footnotetext{
${ }^{1}$ Center for Nanoscience and Engineering, Indian Institute of Science, Bangalore 560012, India. ${ }^{2}$ Physics Department, Indian Institute of Science, Bangalore 560012, India. ${ }^{3}$ Materials Research Center, Indian Institute of Science, Bangalore 560012, India. ${ }^{4}$ Department of Mechanical Engineering and Materials Science, Washington University in St. Louis, Washington, MO 63130, USA. ${ }^{5}$ Material Science and Technology division, Oak Ridge National Laboratory, Tennessee 37831, USA. ${ }^{6}$ Present address: Electrical Engineering, Technion Israel Institute of Technology, Haifa 3200003, Israel. ${ }^{7}$ Present address: Materials Engineering, Technion Israel Institute of Technology, Haifa 3200003, Israel. ${ }^{8}$ Present address: School of Mechanical and Aerospace Engineering, Oklahoma State University, Stillwater 74078 OK, USA. Correspondence and requests for materials should be addressed to S.R. (email: sraghavan@iisc.ac.in)
} 
T he chemical vapor deposition (CVD) of graphene for large area applications is typically carried out by transition metal $(\mathrm{Cu}, \mathrm{Pt}, \mathrm{Co}, \mathrm{Cr}, \mathrm{Rb}$ etc.) catalyzed hydrocarbon (methane, $\mathrm{C}_{2} \mathrm{H}_{4}, \mathrm{C}_{6} \mathrm{H}_{6}$ etc.) decomposition at temperatures in excess of $950^{\circ} \mathrm{C}^{1-6}$. Continuous monolayers are formed on the metal surface by the nucleation and coalescence of graphene domains. Hence, grain boundaries $(\mathrm{GB})$ and triple junctions that are a part of any polycrystalline material are also present in large area monolayer graphene $^{7-9}$. These GB have been extensively studied and their defect density has been theoretically shown to be mainly dependent on the extent of misorientation between the grains ${ }^{10-12}$. However, in CVD graphene, the defect density at a boundary is not determined by the misorientation angle alone. Tsen et al. ${ }^{13,14}$ studied the effect of source gas flow conditions on the quality and type of GB structures formed in CVD grown graphene. Using transmission electron microscopic (TEM) analysis of the boundary along with electrical transport measurements, they reported that the samples grown at higher methane flows had smaller grains, uniform coverage and most importantly, lower GB resistance. Samples grown using lower methane flows were found to have boundaries with overlaps and voids, resulting in higher resistance ${ }^{14}$. The results are markedly important in two aspects. First, the GB defect density was shown to depend not only on the geometric aspects of the grains such as tilt misorientation and edge type, but also on gas flow conditions. Second, electrical transport characterization was found to be an effective tool to inspect the defect density of GB. Their study, however, did not address the correlation between the physico-chemical state of the growth system and defects at GB. More recently, calculations by Dong et al. ${ }^{15}$ showed that one can toggle between covalently bonded boundaries and hydrogen terminated edges by changing hydrogen pressure.

In this article, it is shown that there exists a thermodynamic relationship between the gas phase and defects in the deposited monolayer. Such defects, which are shown to be predominantly located at the domain coalescence boundaries, can be healed and then re-created, by exposing the monolayer to different corresponding carbon potentials. Hence, monolayer properties can be tuned. As an illustration, it is shown that the charge mobility $\left(\mu_{\mathrm{cm}}\right)$ and sheet resistance $\left(R_{\mathrm{s}}\right)$ can be reversibly changed by more than an order of magnitude. This control has allowed us to synthesize graphene with $\mu_{\mathrm{cm}}$ of $20,000 \mathrm{~cm}^{2} \mathrm{~V}^{-1} \mathrm{~s}^{-1}$ in device with dimensions of $100 \mu \mathrm{m} \times 100 \mu \mathrm{m}$. Our results are compared against reported literature in Supplementary Note 1. All these improvements on the monolayer happen in a regime in which Raman measurements ${ }^{16,17}$ are insensitive to the changes occurring in it. Hence, to supplement the electrical transport measurements, water permeation measurement technique is used to identify and spatially locate the incorporated changes in the monolayer. The results highlight the importance of GB closure in obtaining graphene with the best of electronic properties over large areas, in the regime in which the yields of increasing grain size start diminishing (Supplementary Note 2$)^{18}$. In addition, there are many applications that can benefit from the controlled introduction of defects at graphene boundaries. These include the ability to control molecular permeation and sensitivity to various chemicals ${ }^{19,20}$.

\section{Results}

Nucleation, growth, and annealing at constant $\Delta G$. Graphene synthesis using $\mathrm{Cu}$ catalyzed dehydrogenation of methane can be described by the following equation.

$$
\mathrm{CH}_{4} \leftrightarrow \mathrm{C}_{\mathrm{Cu}}+2 \mathrm{H}_{2}
$$

$\mathrm{C}_{\mathrm{Cu}}$ refers to the carbon monolayer (graphene) on the $\mathrm{Cu}$ surface. Equation (1) represents only the thermodynamic terminal points, whereas the internal pathways of the system can be many and are still being debated ${ }^{2}, 21$. The free-energy change driving the growth or supersaturation $(\Delta G)$ is then given by

$$
\Delta G=R T \ln \left(\frac{K}{K^{e q}}\right)
$$

where, $K=P_{\mathrm{CH}_{4}} / P_{\mathrm{H}_{2}}^{2}, K^{e q}$ is the value $K$ at equilibrium, $P_{\mathrm{CH} 4}$, $P_{\mathrm{H} 2}, T$, and $\mathrm{R}$ represent $\mathrm{CH}_{4}$ partial pressure, $\mathrm{H}_{2}$ partial pressure, temperature, and the gas constant, respectively. Details of the experimental conditions used and the resulting supersaturation, calculated using standard formation energies ${ }^{22}$, are given in the Supplementary Note 3 for a range of methane partial pressures. Four groups of samples, $G_{1}-G_{4}$, were studied as summarized in Table 1. The growth details of four specific samples, $S_{1}-S_{4}$, one from each of these groups, to be discussed in this paper are presented in Fig. 1a below and also described in Table 1.

In the schematic representation shown in Fig. 1a, a heat-up stage and $\mathrm{Cu}$ foil anneal stage are used to set the reactor temperature and anneal the substrate, respectively. Supersaturation, $\Delta G=G_{\text {reactants }}-G_{\text {products, }}$, is established in the reactor in the growth stage during which sequential methane dehydrogenation produces a population of adatom species on the $\mathrm{Cu}$ surface. Graphene growth then proceeds through the four stages of incubation, nucleation, growth, and coalescence. Following the 6-minute point, certain samples, see Table 1, are treated with two kinds of post-growth anneal procedures PGA-1 and PGA-2, without breaking vacuum. Finally, the samples are cooled down under the growth condition to room temperature.

\section{Table 1 List of sample types and descriptions}

\section{Sample group type Description}

$\mathrm{G}_{1}$

Standard graphene. Samples grown at a constant $\Delta G$ of $36 \mathrm{~kJ} \mathrm{~mol}^{-1}$ from 0 and up to 14 min. After 6 min a complete monolayer is observed in the SEM and is called visually coalesced graphene (VCG). This 6-min graphene is sample $\mathrm{S}_{1}$. Points corresponding to sample $S_{1}$ are indicated in Fig. 1h, i. Electrical data from sample $S_{1}$ are presented in Supplementary Table 1, row number 5 . Annealed graphene. Samples obtained by first growing up to point VCG at $36 \mathrm{~kJ} \mathrm{~mol}^{-1}$, sample $\mathrm{S}_{1}$ above, and then exposing to $\Delta G$ values higher than $36 \mathrm{~kJ} \mathrm{~mol}^{-1}$ in stage PGA-1 in Fig. 1a without breaking vacuum. Points corresponding to the specific sample $S_{2}$ from this group, which was annealed at $\Delta G$ of $82 \mathrm{~kJ} \mathrm{~mol}^{-1}$, are indicated in Fig. $2 a$, b. Electrical data from sample $S_{2}$ is presented in Supplementary Table 1, row number 14.

$\mathrm{G}_{3}$ Reverse annealed graphene. Samples obtained by first performing growth as in sample $\mathrm{S}_{2}$ above and then exposing to $\Delta G$ values lower than $82 \mathrm{~kJ} \mathrm{~mol}^{-1}$ and down to $36 \mathrm{~kJ} \mathrm{~mol}^{-1}$ in stage PGA-2 in Fig. 1a, without breaking vacuum. Point corresponding to the sample $S_{3}$ from this group, re-annealed at $\Delta G$ of $36 \mathrm{~kJ} \mathrm{~mol}^{-1}$ is indicated in Fig. $2 \mathrm{~b}$. Electrical data from sample $\mathrm{S}_{3}$ is presented in Supplementary Table 1, row number number 18.

$\mathrm{G}_{4}$ Hydrogen-treated graphene. Samples obtained by first performing growth as in sample $\mathrm{S}_{2}$ and then exposing the sample to pure hydrogen ambient, $\Delta G<0$ in PGA-2 without breaking vacuum. Electrical data from sample $S_{4}$ is presented in Supplementary Table 1, row number 19. 

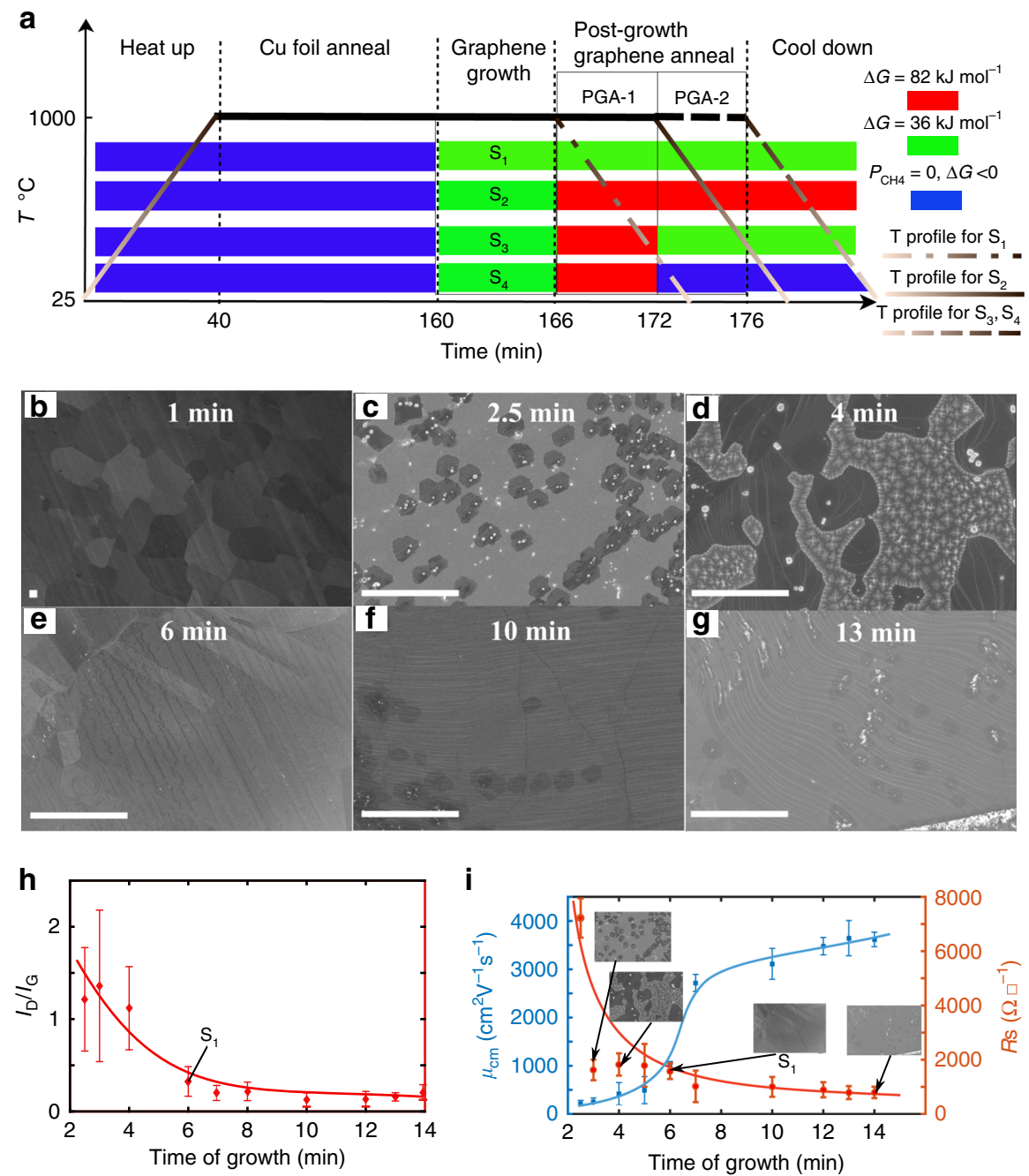

Fig. 1 Growth and characteristics of the reference graphene group $G_{1}$. a Graphene growth conducted in this work and the steps involved are described schematically. The growth atmosphere is color coded and the legend shows the supersaturation conditions. $\mathrm{S}_{1}-\mathrm{S}_{4}$ refer to four different samples as listed in Table 1. b-g SEM images of various stages of graphene growth on $\mathrm{Cu}$ under conditions used for the standard group $\mathrm{G}_{1}$. Growth time (from 160 th minute in Fig. 1a) is indicated in the photographs. $\mathbf{b}$ Incubation period, $t<2 \mathrm{~min}$, in which no nuclei are formed. c Nucleation stage, $t=2.5 \mathrm{~min}$, in which domains of size $\sim 2 \mu \mathrm{m}$ are observed. d Growth stage, $t=4 \mathrm{~min}$, in which individual domains grow by adatom addition until they merge with other similarly growing domains. e Visually coalesced graphene (VCG) observed after 6 min of growth. This sample is referred to as $S_{1}$ and serves as a reference. $\mathbf{f}$ Surface image after $10 \mathrm{~min}$ of growth. $\mathbf{g}$ Surface image after $14 \mathrm{~min}$ of growth. The bright spots are Cu particles that are deposited from the vapor phase during cool down on exposed $\mathrm{Cu}$ areas. $\mathbf{h}$ The time evolution of film defect density as measured by Raman spectroscopy. $\mathbf{i}$ Charge mobility $\left(\mu_{\mathrm{cm}}\right)$ and sheet resistance $\left(R_{\mathrm{s}}\right)$ evolution with growth time. While $\mu_{\mathrm{cm}}$ shows a four-fold change after visual coalescence at 6 min, Raman defect density remains almost unchanged. A Gompertzian-Sigmoidal fit (blue continuous line in i) to the charge mobility is also shown. Scale bars in b-g: $10 \mu \mathrm{m}$

At $\Delta G \leq 32 \mathrm{~kJ} \mathrm{~mol}^{-1}$, graphene grains either did not nucleate or the rate was so slow that no growing domains were observed until a reasonable growth period of $20 \mathrm{~min}$. Upon increasing the $\Delta G$ to $36 \mathrm{~kJ} \mathrm{~mol}^{-1}$, group $\mathrm{G}_{1}$, the surface remained unmodified up to $1 \mathrm{~min}$ as shown in Fig. $1 \mathrm{~b}$. Nuclei of size $\sim 2 \mu \mathrm{m}$ were observed at $2.5 \mathrm{~min}$ as shown in Fig. 1c. The individual grains then grew and started to coalesce. Figure 1d shows a partially covered graphene layer after 4 min of growth. Finally, after about 6 min, the $\mathrm{Cu}$ surface was "completely covered" as can be seen in Fig. 1e. The graphene film after $6 \mathrm{~min}$ of growth will be referred to as "visually coalesced graphene (VCG)" as the SEM micrographs indicate complete coverage and thereafter no further change is detected by SEM. This is sample $S_{1}$. Surface images of $\mathrm{Cu}$ growth surface at 10 mins and 13 mins are shown in figure 1f, $\mathrm{g}$, respectively. The average film defect density extracted from Raman spectroscopic measurements, presented in figure $1 \mathrm{~h}$, (see Supplementary Note 4 for Raman measurement details), also mirrors this temporal evolution. The $\mathrm{I}_{\mathrm{D}} / \mathrm{I}_{\mathrm{G}}$ ratio decreases sharply upto a growth time of $6 \mathrm{~min}$ and then saturates in the period from 6 to $14 \mathrm{~min}$. Thus, it appears from these characterization methods that monolayer deposition is complete by $6 \mathrm{~min}$. Graphene growth by $\mathrm{CVD}$ on $\mathrm{Cu}$ is typically modeled as a self-limiting catalytic process ${ }^{4}$, which can explain the SEM snapshots and $\mathrm{I}_{\mathrm{D}} / \mathrm{I}_{\mathrm{G}}$ saturation behavior. However, as can be noted from Fig. 1i, $\mu_{\mathrm{cm}}$ increases rapidly only after $6 \mathrm{~min}$ of growth-the point at which the monolayer seems complete-and saturates at $13 \mathrm{~min}$. The $\mu_{\mathrm{cm}}$ here is obtained from the constant mobility fit described by Venugopal et al. ${ }^{23}$ as explained in Supplementary Notes 5 and 6 . Kinetic growth saturation has been previously observed in graphene and the properties were shown to have a Gompertziansigmoidal behavior ${ }^{24}$. A Gompertzian fit, shows that $\mu_{\mathrm{cm}}$ increases by a factor of 4 from $1000 \mathrm{~cm}^{2} \mathrm{~V}^{-1} \mathrm{~s}^{-1}$ at $6 \mathrm{~min}$ to saturate at $3600 \mathrm{~cm}^{2} \mathrm{~V}^{-1} \mathrm{~s}^{-1}$ at $14 \mathrm{~min}$. Sheet resistance decreases by a factor of 2 and attains a saturation value of $790 \Omega \square^{-1}$ at 
14 min. These observations belong to the sample set $G_{1}$ in which a constant $\Delta G$ of $36 \mathrm{~kJ} \mathrm{~mol}^{-1}$ was maintained through all stages of growth. This sharp change in electronic properties, beyond the point at which the other structural characterization methods and Raman defect density measurements do not suggest any significant change in the quality of graphene is the first interesting observation. All the electrical measurements, except for samples grown below $4 \mathrm{~min}$ (where the film was not completely coalesced), were performed on $100 \mu \mathrm{m}$ by $100 \mu \mathrm{m}$ graphene devices (See Supplementary Notes 5-7, for more details regarding electrical measurements).

Tuning GB and electron mobility by varying $\Delta G$. Further experiments, sample group $G_{2}$ in Table 1 , were conducted to determine whether $\mu_{\mathrm{cm}}$ and sheet resistance can be further improved with the grain size unchanged. Graphene layers were deposited with $\Delta G$ conditions kept the same as in sample $S_{1}$ in the growth stage (see Fig. 1a). This ensured that the average graphene grain sizes were constant across all samples in this group. Post the VCG stage, these samples were annealed without breaking vacuum for $6 \mathrm{~min}$ with higher $\Delta G$ values up to $112 \mathrm{~kJ} \mathrm{~mol}^{-1}$. A different growth run was used for each value of $\Delta G$ used for annealing (see Supplementary Table 1). This is denoted as PGA-1 in Fig. 1a. At higher $\Delta G$, growth transients were expected to attain the final steady states of interest within 6 min. Hence, a time sequence such as the one in Fig. 1i was not performed for these higher $\Delta G$ values.

The rather dramatic effects of increasing $\Delta G$ on the saturation $\mu_{\mathrm{cm}}$ are shown in Fig. $2 \mathrm{a}$ and sheet resistance is shown in Supplementary Note 6 . The charge mobility $\mu_{\mathrm{cm}}$ increases by 20 fold from 1000 to $20,000 \mathrm{~cm}^{2} \mathrm{~V}^{-1} \mathrm{~s}^{-1}$. The standard deviation in the observed values was $<10 \%$ of the mean values and is shown in the Fig. 2a. Correspondingly, there is a further five-fold reduction in the steady state sheet resistance value to $159 \Omega \square^{-1}$ on raising $\Delta G$ from 36 to $82 \mathrm{~kJ} \mathrm{~mol}^{-1}$. This is the lowest reported sheet resistance thus far (see Supplementary Note 1 and 2 for a compilation of sheet resistances and $\mu_{\mathrm{cm}}$ reported in the literature) in large area CVD graphene and over such large device sizes $(100 \mu \mathrm{m} \times 100 \mu \mathrm{m})$. The Raman $\mathrm{I}_{\mathrm{D}} / \mathrm{I}_{\mathrm{G}}$ ratio again remains flat. Thus, from the VCG stage at which the structural characterization methods seem to have become insensitive to changes in the graphene layer, further processing has thus resulted in a 20 -fold increase in $\mu_{\mathrm{cm}}$. This value is also five-fold higher than the saturation mobility observed under a constant $\Delta G=36 \mathrm{~kJ} \mathrm{~mol}^{-1}$ in $\mathrm{G}_{1}$ (Fig. 1i). The range of sheet resistances achieved here surpass the values obtained in the literature and its comparison is presented in Supplementary Figure 2a.

Additional experiments were performed to determine whether the rise in mobility in Fig. $2 \mathrm{a}$ is reversible. Graphene layers were deposited under conditions that yielded the highest $\mu_{\mathrm{cm}}$ and lowest sheet resistance values (after a treatment under $\Delta G=82 \mathrm{~kJ} \mathrm{~mol}^{-1}$ ), sample $\mathrm{S}_{2}$. Without breaking vacuum these samples were now exposed to decreasing values of $\Delta G$ down to $36 \mathrm{~kJ} \mathrm{~mol}^{-1}$ for $4 \mathrm{~min}$ and $12 \mathrm{~min}$. A different growth run was used for each value of lower $\Delta G$ used during the reversal. It was observed that the difference in $\mu_{\mathrm{cm}}$ values between the 4 -min exposure and the 12-min exposure was within experimental error. Hence, all subsequent exposures to lower $\Delta G$ were limited to $4 \mathrm{~min}$. The results of these measurements are presented in Fig. $2 \mathrm{~b}$. Indeed, the $\mu_{\mathrm{cm}}$ values decrease as $\Delta G$ is lowered in the reactor and trace back the preceding rise almost exactly but for a small hysteresis.

\section{Discussion}

An atomistic picture of the nucleation, growth, and coalescence process of graphene is now discussed to help analyze these mobility results and the ones to be discussed in the next section. It is shown that the reversible phenomena is due to grain boundary thermodynamics and not owing to growth kinetics. From a growth perspective, it is important to establish this fact, as thermodynamic control is more robust than a kinetic one.

The atomistic picture is shown schematically in Fig. 3a. We use the potential of carbon atoms at various locations in the growth environment to first qualitatively explain the observed phenomena. We later use density functional theory (DFT) calculations to support these conclusions. The graphene growth reaction starts by setting a carbon potential in the vapor phase $\left(C_{\mathrm{v}}\right)$ in the form of the methane partial pressure. Methane decomposes on the catalyst and yields a surface carbon potential $\left(C_{s}\right)$. Crystalline graphene domains with a potential $C_{\mathrm{g}}$ (carbon potential within the graphene grain) nucleate and grow by consuming the surface carbon. $C_{\mathrm{s}}$ at any point on the growth surface will vary with time and will be determined by the balance between carbon addition by adsorption and decomposition and carbon removal by desorption, nucleation, and attachment to growing edges. With an increase in coverage, as the domains get closer to form boundaries, the $C_{\mathrm{s}}$ values will get lowered due to reduced catalyst site availability and increased consumption. The reduced $C_{s}$ will lower lateral growth rates and in the extreme case prevent complete coalescence in reasonable time periods. In addition to these three potentials, carbon atoms in the defect structures such as GB,
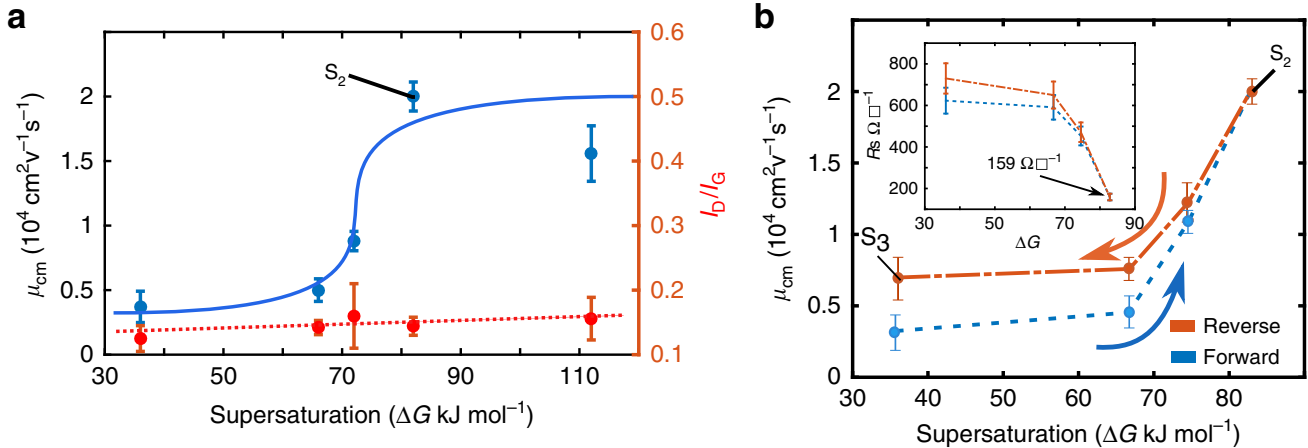

Fig. 2 Reversibility in mobility with reversal in thermodynamic conditions. a Effect of annealing sample $S_{1}$ under increasing supersaturations $(\Delta G)$ on mobility $\left(\mu_{\mathrm{cm}}\right)$ and Raman $\mathrm{I}_{\mathrm{D}} / \mathrm{I}_{\mathrm{G}}$ ratio. $\mu_{\mathrm{cm}}$ is seen to increase significantly with $\Delta G$ and then saturate. The averaged defect density as measured by Raman is insensitive to this change. The sample $S_{2}\left(\Delta G=82 \mathrm{~kJ} \mathrm{~mol}^{-1}\right)$ is indicated. $\mathbf{b}$ Reversal of $\mu_{\mathrm{cm}}$ on reversal of supersaturation, indicating that the phenomenon is thermodynamic in origin and not kinetic. The small hysteresis can be attributed to kinetics. The sheet resistance vs supersaturation and resistance vs gate bias plots are included in the Supplementary Note 6. Samples $S_{2}$ and $S_{3}$ are indicated 
triple junction and line defects in the graphene monolayer will be at higher carbon potentials $\left(C_{\mathrm{d}}\right)$ than $C_{\mathrm{g}}$ and are shown in Fig. 3a as a band.

If $C_{\mathrm{s}}$ became equal to $C_{\mathrm{g}}$ during growth, a coverage saturation will happen despite a global supersaturation $\left(C_{\mathrm{v}}\right.$ being higher than $C_{\mathrm{g}}$ ). If $C_{\mathrm{s}}$ were raised by increasing $C_{\mathrm{v}}$ (increasing $\Delta G$ ), the degree of coverage will increase in a given time of growth. Such growth is termed kinetically controlled and the coverage saturation owing to kinetic limitations has been described before by Celibi et al. using a Gompertzian model ${ }^{24}$. Thus, the increase in mobility with $\Delta G$ or methane partial pressures in Fig. 2a can be attributed to "more faster and complete coverage" in the time frames involved, in the sense that the grain boundary closure is more complete. If this were true, that is, the improvements in mobility in Fig. 2a were purely owing to kinetic reasons as just discussed, then re-annealing the samples that had attained the highest mobilities in lower methane partial pressures $\left(C_{\mathrm{v}}\right.$ still higher than $C_{\mathrm{g}}$ ) should not reverse the mobility gains as observed in Fig. 2b. The reversal is thus proof of the a

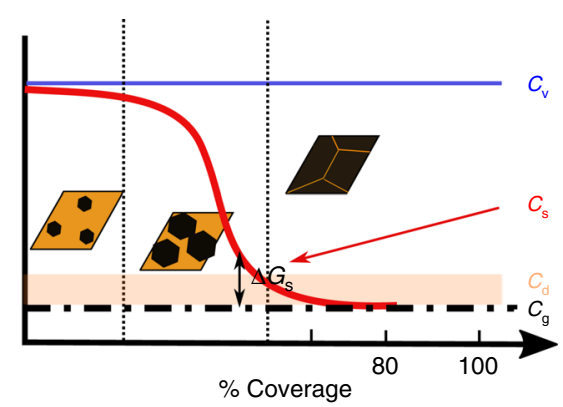

b

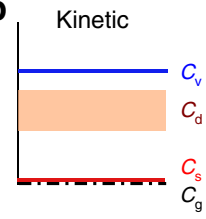

C

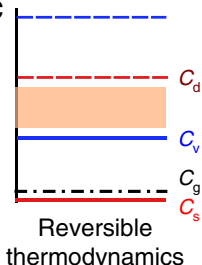

Fig. 3 Schematic of growth thermodynamics and kinetics. a Carbon potentials at various coverage levels with reference to that inside the graphene grain $C_{g} . C_{d}, C_{s}$ and $C_{v}$ represent defect (at grain boundary), surface and vapor phase potentials. $\mathbf{b}$ Energetic situation in which the lack of grain boundary closure will be termed kinetic. Vapor carbon potentials are greater than those at the defect levels and thus closure is not impeded by global thermodynamics. However, local (in the vicinity of the grain boundary being formed) surface potentials determined by adsorptiondesorption kinetics can be lower than the defect potential thus preventing closure. c Energetic situation in which the lack of grain boundary closure is thermodynamic. Those grain boundary structures in which the carbon potentials are higher than $C_{v}$ cannot be formed. Raising carbon potentials will form these structures, as shown by dotted lines, whereas lowering it will remove carbon from these locations leaving behind vacant sites improvements, which happen post grain coalescence, having a thermodynamic origin.

Thermodynamically, the ability to form a particular boundary structure that will complete the coalescence process, will require the global carbon potential, $C_{\mathrm{v}}$ to be greater than $C_{\mathrm{d}}$ as shown in Fig. 3b. If in spite of this scenario it did not form, then the effect is kinetic. Such a situation will arise if $C_{\mathrm{s}}$ determined by the relative rates of adsorption-attachment and desorption were to become lesser than $C_{\mathrm{d}}$. On the other hand, if the situation shown in Fig. $3 \mathrm{~b}$ prevailed, then, thermodynamic constraints will come into play. Those boundary structures that have $C_{\mathrm{d}}>C_{\mathrm{v}}$ will never form as it will not be energetically favorable for carbon from the vapor phase to become part of these defect structures. Increasing/ decreasing $C_{\mathrm{v}}$ will then add/remove carbon from these structures, thus healing/forming defects, vacant carbon sites, reversibly at these locations. The spatial and temporal variation of $C_{\mathrm{s}}$ is difficult to estimate quantitatively. Hence, in the next section we compare the energetics of $C_{\mathrm{v}}$, with that of boundary energies to determine if such an effect is possible.

Dong et al. ${ }^{15}$ have shown that by changing the hydrogen potential in the reactor, one can replace covalently bonded boundaries by hydrogen terminated ones and vice-versa. The experimental work presented here involves changing the carbon potential in the reactor resulting in reversible exchange of carbon atoms between the boundary and the vapor phase. To model this process (See Supplementary Note 8 for the thermochemistry considerations) density functional simulations were carried out to compare the energetic differences between different states of the grain and its boundaries. The thermodynamic reversibility at the grain boundary implies that there are two states, a grain boundary and a defective grain boundary, between which the system can toggle depending on the carbon potential in the system. The defective grain boundary will have to be formed by the breaking of carbon-carbon bonds. It is expected that the resulting dangling bonds will get passivated by hydrogen in the hydrocarbon environment of the reactor. This resultant entity can be approximated by a graphene edge terminated by hydrogen. The reaction that represents this process can be expressed as:

$$
\mathrm{C}-\mathrm{C}^{\prime}-\mathrm{C}+3 \mathrm{H}_{2} \leftrightarrow 2 \mathrm{C}-\mathrm{H}+\mathrm{CH}_{4}
$$

" $\mathrm{C}$ "” represents grain boundary carbon and the equation shows a defect removal and its replacement by an edge. In order to determine whether an edge state with lower energy can exist, two graphene grain boundary configurations and their corresponding edges, Fig. $4 a, b$, were chosen. DFT calculations were performed (see details in Supplementary Note 8) to determine the energies of the grain boundary and the edge. Data from JANAF tables
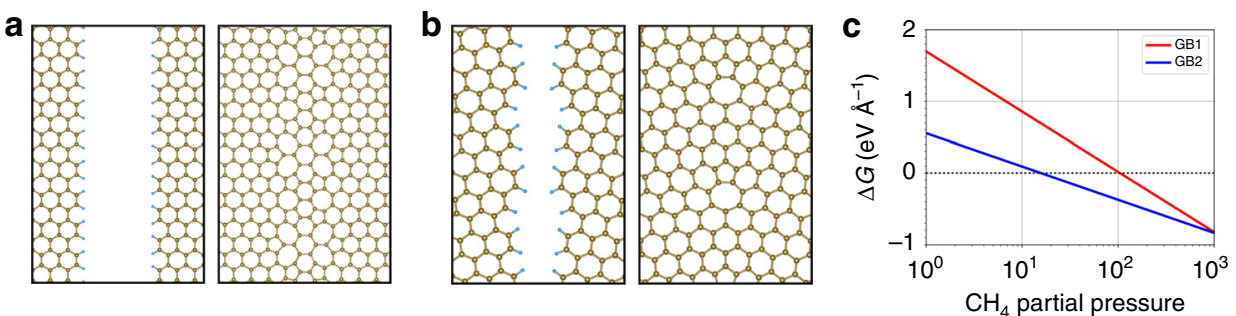

Fig. 4 DFT simulation of graphene grain boundary energetics with methane partial pressure. a, b represent two random mis-orientations GB1 and GB2, respectively. For both, the grain edge passivated with hydrogen, blue atoms, and defect structure on boundary formation are shown. c The free energy change, $\Delta G$, for these two configurations as per equation (3) vs methane partial pressure. A crossover in the plot suggests that depending on the methane partial pressure the reaction can proceed in the forward or backward direction. Increase in pressure favors boundary formation and vice-versa as observed experimentally. The pressure at which it happens depends on the boundary misorientation 
were used to calculate the potentials of hydrogen from $\mathrm{H}_{2}$ and carbon from $\mathrm{CH}_{4}$. These results were then used to calculate the free energy change for the reaction in equation (3) $\left(\Delta G=G_{\text {Reactants }}-G_{\text {Products }}\right)$. The $\Delta G$ variation with methane partial pressure is plotted in Fig. 4c.

A positive value means that the reaction can proceed in the forward direction, i.e., an edge can replace a boundary as it has lower energy. A negative value means that the boundary will be stable against such breakup and defect creation. The plot shows that indeed, a pressure range exists, in which the edge has lower energy than the boundary. There is a crossover after which the reverse is true. The crossover pressure is dependent on the grain boundary configuration. Thus, if at the temperature of graphene growth, there are boundary configurations with positive $\Delta G$, then the phenomenon observed will occur at these sites. This will then result in the thermodynamic reversibility observed.

In the previous discussion, we had hypothesized that vacant carbon sites can be annihilated and created reversibly at GB causing the mobility reversal with change in vapor phase carbon potential. First principles calculations supported this idea. In this section we provide experimental proof. A simple experiment was first performed in which graphene samples with decreasing grain sizes, obtained by increasing supersaturation in the nucleation stage itself (see Supplementary Note 9), were exposed to $5 \mathrm{~min}$ of pure hydrogen at a pressure of 400 Torr and $1000{ }^{\circ} \mathrm{C}$. The resulting change in the sheet resistance before and after hydrogen treatment is shown in Fig. 5. It is clearly seen that under the same hydrogen treatment, which is expected to create defects in the graphene film, samples grown under higher $\Delta G$ and hence smaller grain sizes (or larger grain boundary length per unit area) not only have a larger sheet resistance but also the highest increase on hydrogen annealing. This indicates that the defects are predominantly created at the GB.

In all the four samples previously discussed, $S_{1}-S_{4}$, it was noted that while $\mu_{\mathrm{cm}}$ showed variations, Raman defect density remained mostly unaltered. As Raman defect density is an average value obtained from a large area scan $(10 \mu \mathrm{m} \times 10 \mu \mathrm{m})$, the changes should be happening at length scales that the mapped average does not reflect. To understand the physical picture behind the reversible defect density-mobility trend, a method involving

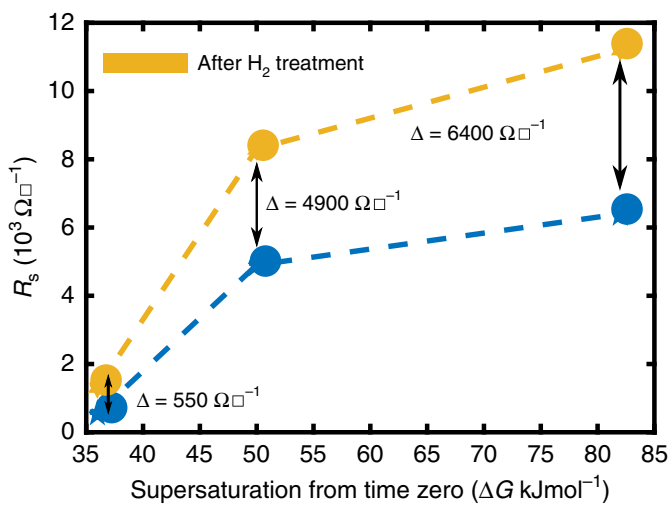

Fig. 5 Effect of grain size on sheet resistance change due to hydrogen treatment. $R_{\mathrm{s}}$ of films nucleated and grown at different supersaturations (See Supplementary Note 9 for effect of supersaturation on grain size) are shown in blue. $R_{\mathrm{s}}$ obtained after a pure hydrogen anneal, or the lowest carbon potential exposure possible, are shown in yellow. Changes in sheet resistance are also marked. Sheet resistance and its change increase with a decrease in grain size, thereby proving that the responsible defects lie at the $G B$ preferential water permeation through defects in graphene followed by etching of an underlying Ge film ${ }^{25,26}$ was employed on the four samples listed in Table 1. This process creates a replica of the defect structure in the graphene monolayer on the underlying $\mathrm{Ge}$ film and renders them optically visible. A detailed time sequence of the etching to be discussed in the following section is presented in the Supplementary Note 10. Optical images are presented in Fig. 6a-h and AFM images in Fig. 6i-l. Figure $6 a$, e show that when the VCG (sample $S_{1}$ ) is examined by this method, the etch pattern formed within just 1 min of permeation, involving $50-100 \mu \mathrm{m}$ sized grains, corresponds to the microstructure of the $\mathrm{Cu}$ substrate on which the monolayer was synthesized (see Fig. 1b). Thus, most of the major defects in VCG lie at the $\mathrm{Cu} G B$. In contrast, in $\mathrm{S}_{2}, \mathrm{~S}_{3}$, and $\mathrm{S}_{4}$, samples that have been annealed in methane post VCG, the $\mathrm{Cu}$ GB are not revealed even after 60 min of permeation (Fig. $6 b-d, f-h$ ). Higher magnification images, second row, show that the etch pattern has an average grain size of $6.7 \mu \mathrm{m}$ (standard deviation of $0.8 \mu \mathrm{m}$ ), which matches well with the values measured using SEM (see Supplementary Note 2). Thus, in these samples the defective regions are predominantly at the coalescence boundaries in the graphene layer.

In addition, in the reverse annealed sample $S_{3}$, the etch pattern lines are thicker (Fig. 6c, g) compared to the annealed sample $\mathrm{S}_{2}$, (Fig. $6 \mathrm{~b}, \mathrm{f}$ ). The AFM scans shown in Fig. 6(i-l) reveal that difference in the surface after permeation experiments in sample $\mathrm{S}_{2}$ and $\mathrm{S}_{3}$. Larger etch pit widths quantitatively correspond to higher rates of permeation ${ }^{25}$ enabled by more defective boundaries. After $100 \mathrm{~min}$ etch, the wider pit depth of $474 \mathrm{~nm}$ in the reverse annealed sample $S_{3}$, when compared to $56 \mathrm{~nm}$ in $\mathrm{S}_{2}$, clearly shows that reverse annealing had indeed made the GB of the monolayer more defective. Further details on the AFM scans are shown in the Supplementary Note 11. Thus, reverse annealing at lower $\Delta G$ has made the graphene GB more defective. One can argue that the reverse annealing might also cause defects within the grain (HRTEM imaging in the next section throws more light on this aspect) that are not detected by the permeability measurements. To determine if this is so, sample $\mathrm{S}_{4}$ was annealed in pure hydrogen at a pressure of 4 Torr. Even in this case as can be seen from Fig. 6d, h only GB are revealed. If the grain were to have become defective, then one will see more uniform etching of the Ge below. Thus, these results are direct evidence for our thesis that the reversible rise and fall in $\mu_{\mathrm{cm}}$, Fig. $2 \mathrm{~b}$, is owing to a decrease/increase in grain boundary defects with a rise/fall in $\Delta G$. The type of defects (structure) and their density along the GB of different samples were inspected using a high resolution TEM. Imaging in a $200 \mathrm{kV}$ TEM was first performed over large areas to establish that the graphene being studied was indeed predominantly a monolayer using tilt diffraction measurements (see Supplementary Note 12). The samples were then observed with an aberration corrected scanning transmission electron microscope (STEM) under a $60 \mathrm{kV}$ electron beam. A large pristine region of the graphene film in $S_{1}$ is shown in Fig. $6 \mathrm{~m}$. The figure shows that the bulk of graphene is mostly defect free with negligible point or line defect density and that the imaging process does not create any noticeable damage to the lattice. Figure $6 \mathrm{n}$ shows a representative grain boundary in $S_{1}$ and Fig. 60 shows a representative boundary in $\mathrm{S}_{4}$. While the boundary in Fig. $6 \mathrm{n}$ shows a string of Stone-Wales defect structures distributed in a region less than $1 \mathrm{~nm}$, that in Fig. 60 shows a region that is at least $2 \mathrm{~nm}$ wide. The grains in Fig. $6 \mathrm{n}$ have a misorientation angle of $13.8^{\circ}$ and the defective atomic columns consist of continuous Stone-Wales (5-7) structures (see Supplementary Note 13 and 14 for details on angular misorientation calculations and hexagon to Stone-Wales ratio calculations). The ratio of these 


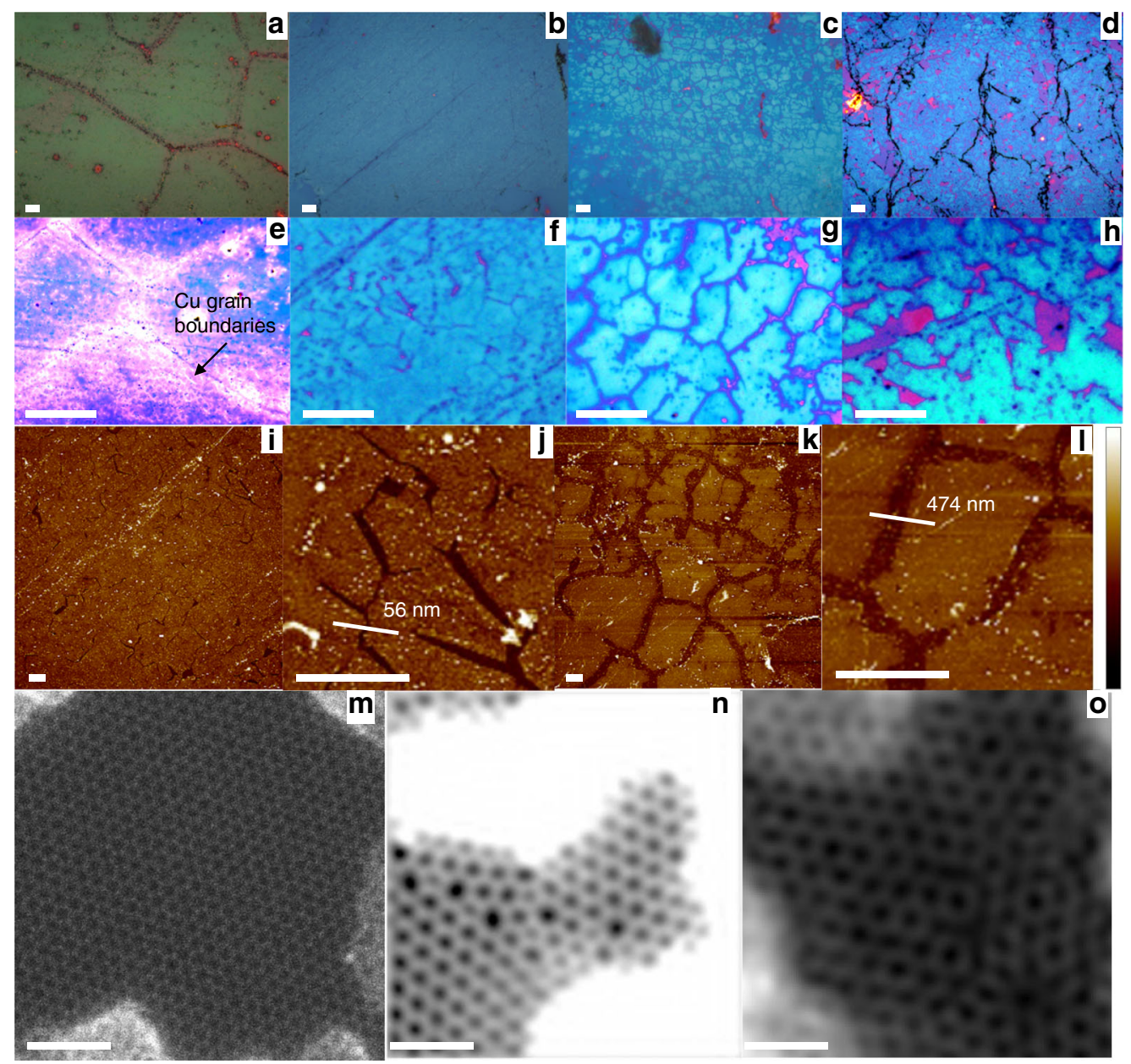

Fig. 6 Visualizing defects. Optical images of Ge underlayer etched by water percolating through defects in the graphene overlayer. a-h Show results after the water percolation experiment on samples $S_{1-4}$ as described in the text. a-d show the $5 \times$ images of the samples $S_{1-4}$, whereas e-h show a higher magnification $(50 \times)$ of the same sequence. Although a-d show large features such as Cu GBs, graphene GBs are clearly visible in $\mathbf{f}-\mathbf{h}$. In sample $\mathrm{S}_{1}$, visually coalesced graphene, the predominant defects lie at the GBs of the Cu foil when it is annealed for $2 \mathrm{~h}$. Grains are $50-100 \mu \mathrm{m}$ in size. In all other cases the defects lie at the coalescence boundaries, 5-10 $\mu \mathrm{m}$ apart, of the graphene monolayer. GBs in $\mathrm{S}_{2}$ annealed at higher supersaturation, are less defective than in $\mathrm{S}_{3}$, as indicated by the higher water percolation rates and hence more deeply etched boundaries. Even annealing in pure hydrogen, $\mathrm{S}_{4}$, only results in defects at the boundary and not within the grain. The purple patches in $\mathbf{a}-\mathbf{h}$ correspond to graphene grains that have flaked off. The pink dots in $\mathbf{h}$ correspond to triple junctions. $\mathbf{i} 40$ micron square AFM scan of surface of $S_{2}$ after the percolation experiment. $\mathbf{j} 7$ micron square AFM scan of surface of $S_{2}$ after permeation experiment with line scan to show etch pit width. $\mathbf{k} 40$ micron square AFM scan of surface of $S_{3}$ after the percolation experiment. 17 micron square AFM scan of surface of $S_{3}$ after percolation experiment with line scan to show etch width. $\mathbf{m}$ Shows a pristine graphene lattice without defects in $S_{1}$. $\mathbf{n}$ Shows a representative boundary in sample $S_{1}$ with a tilt misorientation of $13.86^{\circ}$. o Representative boundary in sample $S_{4}$ with a misorientation of $18^{\circ}$. Image contrast adjusted for better visibility. Scale bars: a-h $10 \mu \mathrm{m}$; i-l: $3 \mu \mathrm{m}$ and $\mathbf{m}-\mathbf{0}: 1 \mathrm{~nm}$. Color bar for i-l: $-20 \mathrm{~nm}$ to $20 \mathrm{~nm}$

structures to those of the standard hexagon is $>1$. Theoretical predictions based on geometric considerations (Supplementary Note 14) predict a ratio of $<0.6$ for a misorientation of $\sim 13.8^{10}$. Thus, the higher defect density at the boundary in $S_{1}$, the $6 \mathrm{~min}$ VCG, is indicative of a graphene that has not been fully formed. The triple junction that is close to the imaged boundary can also influence the defect density. However, what this simple calculation nevertheless shows is that defect densities at boundaries and especially closer to triple junctions will be much larger than those theoretically anticipated. They in turn can have a significant impact on the properties of the monolayer. On the other hand, Fig. 6o shows a boundary, which is both wider and higher in defect density than the one in Fig. 6n. Voids are seen at the boundary in addition to standard Stone-Wales defect sites clearly showing the effect of annealing at lower $\Delta G$. This explains the lower $\mu_{\mathrm{cm}}$ (see Supplementary Table 1) and high water permeability in $S_{4}$. Only covalently bonded GB were observed at the supersaturations used in the work done as part of this research as can be noted from the SEM and STEM images. Overlapped boundaries as reported by other groups ${ }^{15}$ were not observed under the conditions used for synthesizing the samples used in this paper (Supplementary Note 3). However, at much lower methane partial pressures and lower growth rates, overlapped GB were also observed. A discussion of the characteristics of those samples is beyond the scope of this paper.

In summary, we show that the configuration of grain boundary defects in graphene monolayers have a thermodynamic relationship with carbon potential in the vapor phase. These defects can be created and healed reversibly. This relationship allows one to control grain boundary structure. The resulting large variation in grain boundary resistance allows the field effect mobility in graphene monolayers to be changed by more than an order of magnitude, from $1000 \mathrm{~cm}^{2} \mathrm{~V}^{-1} \mathrm{~s}^{-1}$ to $20,000 \mathrm{~cm}^{2} \mathrm{~V}^{-1} \mathrm{~s}^{-1}$ and 
sheet resistance from 1200 to $159 \Omega \square^{-1}$, the best reported yet for such large device sizes. Most of these microstructural changes that result in such large variations in electrical properties cannot be detected by standard Raman and SEM characterization. The study also shows that GB might be equally, if not more important, than grain size in graphene.

\section{Methods}

CVD graphene growth. Graphene was grown in a custom-made CVD reactor ${ }^{22,27}$. Ultra-pure (99.9999\%) hydrogen, and nitrogen flowing through a purifier, $99.98 \%$ pure methane and $99.98 \%$ pure $\mathrm{Cu}$ from Sigma Aldrich were used for synthesis Although growth was done at $1000^{\circ} \mathrm{C}$, reactor chemistry was changed to vary carbon potential by varying methane and hydrogen flows. Gas flows for the supersaturation used in this article are shown in Supplementary Fig. 3. Total reactor pressure was maintained at 4 Torr for all our experiments. Such a condition was chosen to have $>85 \%$ monolayer coverage. A wet transfer process as described elsewhere ${ }^{27}$ was used to transfer graphene onto a $285 \mathrm{~nm}$ thermally grown silica/ heavily doped $p$-type Si substrate.

Each data point in Figs. 1i, 2a, b, and 5 were generated by measurements on monolayers grown for that specific purpose without breaking vacuum from start to finish as explained in the text. The standard deviations were calculated from measurements made on at least four devices from a growth run. The growth experiments were also repeated to check the result consistency. The information about the samples and their details are provided in the list of samples provided in Supplementary Table 1 .

Raman measurements. Raman spectroscopic measurements on these transferred layers were carried out with a LabRAM HR spectrometer fitted with a $532 \mathrm{~nm}$ laser source. The instrument was fitted with precision micrometer stage for mapped measurements. Further details on Raman spectroscopic measurements are provided in Supplementary Note 4 .

Electrical measurements. Van der Pauw devices for sheet resistance measurements and MOS FET structures for mobility measurements were fabricated using standard optical lithographic techniques and the graphene channel was defined using a mild oxygen plasma etch using an Oxford reactive ion etcher. Except for electrical measurements below $4 \mathrm{~min}$ in Fig. $1 \mathrm{i}$ all the electrical measurements were made on $100 \mu \mathrm{m} \times 100 \mu \mathrm{m}$ area as shown in Supplementary Note 5 . The measurement statistics and fitting examples are also provided in Supplementary Note 6. For the samples grown for less than $4 \mathrm{~min}$, the graphene film was not continuous and hence, electron beam lithographically made devices were used. Details are provided in Supplementary Note 7. All electrical measurements were made after current annealing the sample under a current density of $3 \times 10^{8} \mathrm{~A} / \mathrm{cm}^{2}$ for $3 \mathrm{~min}$ under a pressure of $4 \times 10^{-5} \mathrm{mbar}$ of air. Given that the average grain size is $\sim 7.08$ $\mu \mathrm{m}$ (standard deviation of $0.88 \mu \mathrm{m}$ ), (See Supplementary Fig. 2) the $100 \mu \mathrm{m}$ channel widths are expected to yield average values that represent electronic properties over large areas.

Etch measurements and AFM. Ge etch experiments to characterize defects, developed in our group ${ }^{25}$, were conducted using an Olympus made immersion lens setup fitted with an Olympus optical acquisition system at room temperature and in open air. The etch profile measurements ${ }^{25}$ were performed using a Bruker made atomic force microscope.

TEM. Diffraction measurements were performed using a FEI T20 transmission electron microscope at $200 \mathrm{kV}$ and the STEM measurements were made using an aberration corrected Nion Ultra STEM 100 microscope operated at $60 \mathrm{KV}$.

\section{Data availability}

The data that support the findings of this study are available from the corresponding author upon reasonable request.

Received: 27 May 2018 Accepted: 4 February 2019

Published online: 06 March 2019

\section{References}

1. Li, X. et al. Large-area synthesis of high-quality and uniform graphene films on copper foils. Science 324, 1312-1314 (2009).

2. Mattevi, C., Kim, H. \& Chhowalla, M. A review of chemical vapour deposition of graphene on copper. J. Mater. Chem. 21, 3324-3334 (2011).

3. Kidambi, P. R. et al. Observing graphene grow: catalyst--graphene interactions during scalable graphene growth on polycrystalline copper. Nano. Lett. 13, 4769-4778 (2013).
4. Kim, H., Saiz, E., Chhowalla, M. \& Mattevi, C. Modeling of the self-limited growth in catalytic chemical vapor deposition of graphene. New J. Phy. 15, 053012 (2013)

5. Yan, Z. et al. Toward the synthesis of wafer-scale single-crystal graphene on copper foils. ACS Nano 6, 9110-9117 (2012).

6. Sun, Z. et al. Growth of graphene from solid carbon sources. Nature $\mathbf{4 6 8}$, 549-552 (2010)

7. Huang, P. Y. et al. Grains and grain boundaries in single-layer graphene atomic patchwork quilts. Nature 469, 389-392 (2011)

8. Yazyev, O. V. \& Chen, Y. P. Polycrystalline graphene and other twodimensional materials. Nat. Nanotechnol. 9, 755-767 (2014).

9. Banhart, F., Kotakoski, J. \& Krasheninnikov, A. V. Structural defects in graphene. ACS nano 5, 26-41 (2010).

10. Yazyev, O. V. Polycrystalline graphene: atomic structure, energetics and transport properties. Solid State Commun. 152, 1431-1436 (2012).

11. Yazyev, O. V. \& Louie, S. G. Electronic transport in polycrystalline graphene. Nat. Mater. 9, 806-809 (2010).

12. Clark, K. W. et al. Spatially resolved mapping of electrical conductivity across individual domain (grain) boundaries in graphene. ACS Nano 7, 7956-7966 (2013).

13. Tsen, A. W., Brown, L., Havener, R. W. \& Park, J. Polycrystallinity and stacking in CVD graphene. Acc. Chem. Res. 46, 2286-2296 (2013).

14. Tsen, A. W. et al. Tailoring electrical transport across grain boundaries in polycrystalline graphene. Science 336, 1143-1146 (2012).

15. Dong, J. et al. Formation mechanism of overlapping grain boundaries in graphene chemical vapor deposition growth. Chem. Sci. 8, 2209-2214 (2017).

16. Ferrari, A. C. \& Robertson, J. Interpretation of Raman spectra of disordered and amorphous carbon. Phys. Rev. B 61, 14095-14107 (2000).

17. Ferrari, A. C. Raman spectroscopy of graphene and graphite: disorder, electron-phonon coupling, doping and non adiabatic effects. Solid State Commun. 143, 47-57 (2007).

18. Isacsson, A. et al. Scaling properties of polycrystalline graphene: a review. $2 D$ Mater. 4, 012002 (2016)

19. Kumar, S., Pratap, R. \& Raghavan, S. Ultrahigh fluid diffusivity in graphenelined nanochannels. Appl. Phys. Lett. 108, 091606 (2016).

20. Colombo, L., Wallace, R. M. \& Ruoff, R. S. Graphene growth and device integration. Proc. IEEE 101, 1536-1556 (2013).

21. Liao, M. S., Au, C. T. \& Ng, C. F. Methane dissociation on Ni, Pd, Pt and Cu metal (111) surfaces - a theoretical comparative study. Chem. Phys. Lett. 272, 445-452 (1997).

22. Ghosh, P. et al. Insights on defect-mediated heterogeneous nucleation of graphene on copper. J. Phys. Chem. C. 119, 2513-2522 (2015).

23. Venugopal, A. et al. Effective mobility of single-layer graphene transistors as a function of channel dimensions. J. Appl. Phys. 109, 104511 (2011).

24. Celebi, K. et al. Evolutionary kinetics of graphene formation on copper. Nano. Lett. 13, 967-974 (2013).

25. Suran, S., Bharadwaj, K., Raghavan, S. \& Varma, M. M. Bright-field nanoscopy: visualizing nano-structures with localized optical contrast using a conventional microscope. Sci. Rep. 6, 25011 (2016).

26. Suran, S., Balasubramanian, K., Raghavan, S. \& Varma, M. M. Spatially resolved observation of water transport across nanomembranes using brightfield nanoscopy. Appl. Phys. Lett. 113, 043701 (2018).

27. Balasubramanian, K. B., Pratap, R. \& Raghavan, S. Transfer free suspended graphene devices on silicon using electrodeposited copper. J. Vac. Sci. Technol. B 32, 010603 (2014).

\section{Acknowledgements}

We acknowledge the National Nano Fabrication center (NNFC) and the Micro/Nano characterization facility (MNCF) for the fabrication and characterization work reported in this article. These centers are supported by grants from the Ministry of Electronics and Information Technology, Department of Science and Technology under NNetRA and the Ministry of Human Resources Development. We also acknowledge the financial support of Department of Science and Technology, Government of India under the Grant No SR/ S2/CMP-02/2007 and the Thematic Unit of Excellence for Nano Science and Technology project. We thank professor N. Ravishankar and professor Arindam Ghosh for insightful discussions. A part of this work was conducted at the Center for Nanophase Materials Science, DOE Office of Science User Facility. RM was supported by National Science Foundation DMREF grant 1729787.

\section{Author contributions}

TB and MJ conducted the first-principle studies, RS, RM, and PG performed the highresolution TEM studies. KB fabricated the device for water permeation studies, and SS and $\mathrm{MV}$ conducted the permeation experiment. $\mathrm{KB}$ and $\mathrm{AM}$ fabricated the devices for electrical studies, and $\mathrm{KB}$ performed the electrical transport studies. $\mathrm{KB}, \mathrm{RP}$, and $\mathrm{SR}$ designed the experiments and wrote the manuscript. All the authors contributed for discussions and analysis leading to the article. 


\section{Additional information}

Supplementary Information accompanies this paper at https://doi.org/10.1038/s41467019-09000-8

Competing interests: The authors declare no competing interests.

Reprints and permission information is available online at http://npg.nature.com/ reprintsandpermissions/

Journal peer review information: Nature Communications thanks Feng Ding and the other anonymous reviewers for their contribution to the peer review of this work.

Publisher's note: Springer Nature remains neutral with regard to jurisdictional claims in published maps and institutional affiliations. (c) (i) Open Access This article is licensed under a Creative Commons Attribution 4.0 International License, which permits use, sharing, adaptation, distribution and reproduction in any medium or format, as long as you give appropriate credit to the original author(s) and the source, provide a link to the Creative Commons license, and indicate if changes were made. The images or other third party material in this article are included in the article's Creative Commons license, unless indicated otherwise in a credit line to the material. If material is not included in the article's Creative Commons license and your intended use is not permitted by statutory regulation or exceeds the permitted use, you will need to obtain permission directly from the copyright holder. To view a copy of this license, visit http://creativecommons.org/ licenses/by/4.0/.

(C) The Author(s) 2019 